\title{
Colony-stimulating factor 1 potentiates lung cancer bone metastasis
}

\author{
Jaclyn Y Hung ${ }^{1}$, Diane Horn ${ }^{2}$, Kathleen Woodruff ${ }^{2}$, Thomas Prihoda ${ }^{2}$, Claude LeSaux ${ }^{3}$, Jay Peters ${ }^{4}$, Fermin Tio ${ }^{2}$ and \\ Sherry $L$ Abboud-Werner ${ }^{2}$
}

Colony-stimulating factor 1 (CSF1) is essential for osteoclastogenesis that mediates osteolysis in metastatic tumors. Patients with lung cancer have increased CSF1 in serum and high levels are associated with poor survival. Adenocarcinomas metastasize rapidly and many patients suffer from bone metastasis. Lung cancer stem-like cells sustain tumor growth and potentiate metastasis. The purpose of this study was to determine the role of CSF1 in lung cancer bone metastasis and whether inhibition of CSF1 ameliorates the disease. Human lung adenocarcinoma A549 cells were examined in vitro for CSF1/CSF1R. A549-luc cells were injected intracardiac in NOD/SCID mice and metastasis was assessed. To determine the effect of CSF1 knockdown (KD) in A549 cells on bone metastasis, cells were stably transfected with a retroviral vector containing short-hairpin CSF1 (KD) or empty vector (CT). Results showed that A549 cells express CSF1/CSF1R; CSF1 increased their proliferation and invasion, whereas soluble CSF1R inhibited invasion. Mice injected with A549-luc cells showed osteolytic bone lesions 3.5 weeks after injection and lesions increased over 5 weeks. Tumors recapitulated adenocarcinoma morphology and showed osteoclasts along the tumor/bone interface, trabecular, and cortical bone loss. Analyses of KD cells showed decreased CSF1 protein levels, reduced colony formation in soft agar assay, and decreased fraction of stem-like cells. In CSF1 KD mice, the incidence of tumor metastasis was similar to controls, although fewer CSF1KD mice had metastasis in both hind limbs. KD tumors showed reduced CSF1 expression, Ki-67+ cells, and osteoclasts. Importantly, there was a low incidence of large tumors $>0.1 \mathrm{~mm}^{2}$ in CSF1KD mice compared with control mice (10\% vs $62.5 \%$ ). This study established a lung osteolytic bone metastasis model that resembles human disease and suggests that CSF1 is a key determinant of cancer stem cell survival and tumor growth. Results may lead to novel strategies to inhibit CSF1 in lung cancer and improve management of bone metastasis.

Laboratory Investigation (2014) 94, 371-381; doi:10.1038/labinvest.2014.1; published online 27 January 2014

KEYWORDS: bone; cancer; CSF1; lung; metastasis

Lung cancer is the leading cause of cancer deaths world wide. ${ }^{1}$ The majority of lung cancers are non-small-cell type and, of those, adenocarcinoma is the most common. These tumors have a high mortality rate and metastasize rapidly, often within months of diagnosis, with $60-70 \%$ of patients developing bone metastasis., ${ }^{2,3}$ Lesions are predominantly osteolytic, involving vertebrae, rib, pelvis, and femur that lead to significant morbidity from spinal cord compression, pathologic fractures, and intractable pain. Metastasis to bone is associated with a poor prognosis and a mean survival of 9.7 months. ${ }^{2}$
Colony-stimulating factor 1 (CSF1) is essential for osteoclastogenesis that mediates osteolysis in metastatic tumors to bone. The effect of CSF1 is mediated via the $c$-fms tyrosine kinase receptor (CSF1R) that is expressed on mononuclear phagocytes, osteoclasts, and some cancer cells. ${ }^{4-7}$ Abnormally high CSF1 and/or CSF1R have been associated with aggressive behavior of malignancies, including breast, prostate, and ovarian cancer. ${ }^{6,7}$ The importance of CSF1 in cancer metastasis has been shown in humans with lung cancer and in rodent models. In rodents with ovarian or breast cancer, overexpression of CSF1 accelerated tumor progression and

\footnotetext{
${ }^{1}$ Division of Hematology and Oncology, Department of Pediatrics, Greehey Children's Cancer Research Institute, University of Texas Health Science Center, San Antonio, TX, USA; ${ }^{2}$ Department of Pathology, University of Texas Health Science Center at San Antonio and South Texas Veterans Health Care System, San Antonio, TX, USA; ${ }^{3}$ Division of Cardiology, University of Texas Health Science Center at San Antonio, San Antonio, TX, USA and ${ }^{4}$ Department of Pulmonary Medicine, University of Texas Health Science Center at San Antonio and South Texas Veterans Health Care System, San Antonio, TX, USA

Correspondence: Dr SL Abboud-Werner, MD, Department of Pathology, University of Texas Health Science Center at San Antonio and South Texas Veterans Health Care System, 7703 Floyd Curl Drive, San Antonio, TX 78229, USA.
}

E-mail: abboudwerner@uthscsa.edu

Received 7 June 2013; revised 22 November 2013; accepted 22 December 2013 
metastasis. ${ }^{8,9}$ Patients with lung cancer show increased circulating CSF1 and high levels correlate with tumor progression and poor prognosis. ${ }^{10}$ Lung cancer cells including the human A549 lung adenocarcinoma cell line express CSF1R, CSF1 mRNA, and protein, and, in vitro, CSF1 increases A549 proliferation and invasion. ${ }^{11-13}$ Studies indicate that stemlike cells within the tumor, referred to as 'cancer stem-like cells' (CSCs), sustain tumor growth, promote metastasis, and have a role in resistance to chemotherapy and relapse. ${ }^{14,15} \mathrm{We}$ have shown that human lung cancers and A549 cells contain a small number of CSCs that are detected as a side population (SP) on fluorescence-activated cell-sorting (FACS) analysis. ${ }^{16}$ In xenograft experiments, SP cells isolated from A549 cells showed increased invasion and tumorigenicity compared with the non-SP.

A major impediment to understanding the biology of lung cancer bone metastasis has been the lack of animal models. Conventional rodent models of lung cancer rarely metastasize and the cells of origin are not well defined. ${ }^{17}$ We and others have shown that CSF1 deficiency in op/op or CSF1 knockout mice decreases osteoclasts and bone resorption. ${ }^{18,19}$ Few studies have described bone metastasis models related to lung cancer and none have examined CSF1 in this model or in relation to CSCs. We hypothesized that knockdown (KD) of CSF1 in tumor cells would reduce the incidence and progression of bone metastasis and osteoclastic bone destruction. The aims of the present study were to characterize the A459 lung cancer bone metastasis model and determine whether inhibition of CSF1 in tumor cells affects CSCs and ameliorates the onset and/or progression of metastasis. Results showed that CSF1KD in A549 cells decreased SP cells and anchorage-independent growth in vitro and was associated with decreased tumor progression in mice. These findings may be highly relevant for clinical lung cancer where CSF1 levels are elevated and CSCs are implicated in disease progression.

\section{MATERIALS AND METHODS RT-PCR and CSF1 protein assay}

A549 cells were cultured in Dulbecco's modified Eagle's medium (DMEM, Gibco, Life Technologies ${ }^{\mathrm{TM}}$ ) supplemented with $10 \%$ fetal calf serum (FCS) and incubated at $37^{\circ} \mathrm{C}$ in $5 \% \mathrm{CO}_{2}$. Total RNA was isolated using TRIzol reagent (Life Technologies ${ }^{\mathrm{TM}}$ ) and subjected to RT-PCR using specific primers sets for human CSF1 and CSF1R (30 pmol each) as previously described. ${ }^{7,20}$ To determine CSF1 protein production, A549 cells grown to $80 \%$ confluence were placed in serum-free (SF) medium and, at the indicated time points, cell-free conditioned medium was assayed for CSF1 using an ELISA that specifically detects human CSF1 (R\&D Systems).

\section{Matrigel Invasion Assay}

The effect of CSF1 on the invasive potential of A549 cells was determined using 24-well Matrigel invasion chambers (BD
Biosciences). Matrigel matrix serves as a reconstituted basement membrane in vitro. Cells were seeded into upper inserts at $5 \times 10^{4}$ per insert in SF DMEM. The outer wells were filled with SF medium with or without CSF1 $(200 \mathrm{ng} / \mathrm{ml})$ as a chemoattractant. In some wells, soluble CSF1R $(2.5 \mu \mathrm{g} / \mathrm{ml}$, $\mathrm{R} \& \mathrm{D}$ Systems) was added to the upper insert. After $48 \mathrm{~h}$, the non-invading cells were removed by swabbing the top layer of Matrigel with a Q-tip. The membrane containing invading cells was stained with hematoxylin and cells were counted. Data are expressed as cells invaded through Matrigel normalized to the control, which was arbitrarily set at 1.0 .

\section{Mice and Intracardiac Injection}

The A549-luc cell line, constitutively expressing the luciferase (luc) reporter gene, was kindly provided by Dr Jerry Shay (University of Texas Southwestern Medical School). ${ }^{21}$ Subconfluent cells were fed with fresh medium $24 \mathrm{~h}$ before injection, then harvested, and assessed for viability using trypan blue. Tumor cells $\left(5 \times 10^{5}\right.$ viable cells in $100 \mu$ l of PBS) were injected with a $27-\mathrm{G}$ needle into the left cardiac ventricle of 5- to 8-week-old NOD/SCID mice (Charles Rivers, Wilmington, MA, USA) as previously described. ${ }^{22}$ Mice were monitored weekly for onset of bone metastasis and tumor progression using whole-body bioluminescent imaging (BLI). ${ }^{23}$ D-Luciferin substrate $(150 \mathrm{mg} / \mathrm{kg})$ was injected intraperitoneally $10 \mathrm{~min}$ before imaging. Light emission from the tissue regions (relative photons/second) was measured using Living Image software (Xenogen). Relative intensities of light emission were represented as a pseudocolor image ranging from blue (least intense) to red (most intense). In subsequent experiments, $1 \times 10^{5}$ cells were injected in CSF1KD and control mice. Intracardiac injection was successful in $50-60 \%$ of experimental mice. Mice showing tumor masses in the heart and/or in the lung because of improper needle placement were excluded from the study. Animal protocols were approved by the Institutional Animal Care and Use Committee at the University of Texas Health Science Center.

\section{$\mathrm{X}$-ray and microCT imaging}

Areas of osteolytic bone metastasis, identified as radiolucent lesions, were detected in hind limbs and vertebrae of mice using a MX-20 Faxitron unit (Faxitron Bioptics, Lincolnshire, IL, USA). Quantification of lesion number and area was performed using the Bioquant Imaging System (Bioquant Image Analysis, Nashville, TN, USA). Lesions observed by radiographs were confirmed by histology. Hind limbs were fixed in 10\% formalin and, before histology, placed in $70 \%$ ethanol for microCT analysis. MicroCT images were acquired using a high-resolution scanner (Bruker 1172, Kontich, Belgium) at the following settings: $60 \mathrm{kV}$ energy, $167 \mu \mathrm{A}$ beam intensity, $0.5 \mathrm{~mm}$ aluminum filter, $0.7^{\circ}$ rotation step, 4 frame average, and $10 \mu \mathrm{m}$ pixel size. 


\section{Bone Histology and Immunohistochemistry}

Bones were decalcified in 14\% EDTA solution, embedded in paraffin, and 5- $\mu \mathrm{m}$-thick sections were cut along the midsagittal plane and stained with hematoxylin and eosin. Adjacent sections were examined in parallel for tumor burden, tartrate-resistant acid phosphatase, and immunohistochemical stains. Histomorphometric measurements of tumor burden and osteolysis were performed using the Bioquant Osteo II software and included: total tumor volume; total trabecular and cortical bone volume; and osteoclast number $(\#) / \mathrm{mm}$ of tumor/bone interface (denoted as $\mathrm{OC} / \mathrm{mmBS}$ ). Total tumor volume was measured in the femur and tibia of both hind limbs. Total trabecular bone volume was measured from the growth plate down $1.5 \mathrm{~mm}$ in both femur and tibia; cortical bone volume was also calculated in this region. The number of osteoclasts along the tumor/bone interface in experimental mice was counted in five random fields $(x 400$ magnification) and compared with osteoclasts/mm BS in control mice.

Immunohistochemistry was performed using primary antibodies: mouse anti-human cytokeratin AE1/AE3 (7602135, Ventana Medical Systems, Tucson, AZ, USA), rabbit antihuman CSF1 (1:100, sc-13103, Santa Cruz, Biotechnology, Santa Cruz, CA, USA), rabbit anti-human Ki-67 (790-4286, Ventana Medical Systems), rat anti-mouse F4/80 (1:100, MCA497, AbD Serotec, Raleigh, NC, USA), and rat antimouse CD31 (1:100, CM303B, Biocare Medical, Concord, CA, USA) and a standard HRP-polymer detection kit (Biocare Medical). Apoptotic cells were detected using TdTmediated dUTP nick-end labeling (TUNEL) as previously described. ${ }^{18}$ CSF1 protein expression was analyzed in a $400 \times 400 \mu \mathrm{m}$ region of interest in four random fields and calculated as the integrated optical density. For Ki-67, positive and negative tumor cells were counted in five random fields and the percentage of Ki-67-positive cells was calculated. F4/80-positive macrophages and C31-positive vessels were quantified within the tumor bed, excluding the periphery, and data are expressed as macrophages or vessels per $\mathrm{mm}^{2}$ tumor area.

\section{CSF1KD and Flow Cytometry}

A549 cells were stably transfected with $1 \mu \mathrm{g}$ of a retroviral vector containing either a 29-mer short-hairpin (sh)CSF1 construct or a empty vector control (pGFP-V-RS; OriGene Technologies). The SP was identified based on their ability to efflux Dye Cycle Violet, which on FACS analysis appears as a distinct population of cells on the side of the FACS profile. ${ }^{16}$ Briefly, cells were resuspended at $1 \times 10^{6}$ in DMEM with $2 \%$ FCS and $10 \mathrm{mM}$ HEPES buffer. Dye Cycle violet reagent, Vybrant ${ }^{\circledR}$ DyeCycle ${ }^{\mathrm{TM}}$ Violet stain (DCV, Molecular Probes, Life Technologies ${ }^{\mathrm{TM}}$ ), was added at a final concentration of $5 \mu \mathrm{g} / \mathrm{ml}$ in the presence or absence of reserpine $(50 \mu \mathrm{M})$ and cells were incubated at $37^{\circ} \mathrm{C}$ for $90 \mathrm{~min}$. Cells were then washed with ice-cold Hank's Balanced Salt Solution with $2 \%$ FCS and $10 \mathrm{mM}$ HEPES. Propidium iodide $(2 \mu \mathrm{g} / \mathrm{ml})$ was added to the cells to gate viable cells and analysis was performed using a FACS Canto (Becton Dickinson). The DCV reagent was excited at $405 \mathrm{~nm}$ and its fluorescence was dual-wavelength analyzed (blue, $406-446 \mathrm{~nm}$; red, 650 $670 \mathrm{~nm}$ ). Data were expressed as the percent of viable cells gated as SP normalized to the control, which was arbitrarily set at $100 \%$.

\section{Cell Proliferation Assay}

The effect of CSF1KD on cell proliferation was examined by cell counting experiments. Subconfluent CSF1KD and control cells were plated at a concentration of $2 \times 10^{4}$ cells/well in 12-well dishes in complete medium and incubated for 2 and 5 days. At each time point, cells were trypsinized and counted.

\section{Anchorage-Independent Growth Assay}

The effect of CSF1KD on anchorage-independent growth of A549 cells was assessed using the Cytoselect ${ }^{\mathrm{TM}}$ 96-Well Transformation Assay (CellBiolabs, San Diego, CA, USA). Cells were seeded at both $5 \times 10^{3}$ cells/well and $1 \times 10^{4}$ cells/ well in a 96-well plate containing complete medium with $1.2 \%$ agar. Fresh medium was replaced every 2 days. After 7 days, cells were lysed, stained with CyQuant:GR dye working solution and colony formation was quantified by measuring the fluorometric detection using the GloMax ${ }^{\circledR}$-Multi Microplate Multimode Reader (Promega). Three independent experiments were performed. The data are expressed as the percent colony formation in CSF1KD cells relative to the control, with the control arbitrarily set at 100\%.

\section{Statistical Analysis}

The means and standard errors (s.e.) of all numeric data were calculated. Data were analyzed statistically using the unpaired Student's $t$-test or a trimmed non-parametric test when appropriate. Differences were considered statistically significant for comparisons of data sets yielding $P \leq 0.05$. Analysis of mice with control and CSF1KD tumors was statistically significant $P \leq 0.05$ by Fisher's exact test for $2 \times 3$ tables or the exact $\chi^{2}$ test.

\section{RESULTS \\ A549 cells Express CSF1 and CSF1R, and CSF1 Enhances Invasion}

To examine the gene expression of CSF1 and CSF1R in the A549-luc cell line used in our studies, total RNA was subjected to RT-PCR analysis using specific primer sets as previously described. ${ }^{12,13,20}$ Figure 1a shows that A549 cells express the 322-bp CSF1 transcript and the 360-bp CSF1R transcript similar to control breast cancer cell lines. The level of CSF1 protein expression was assessed using ELISA. CSF1 protein production by A549 cells increased in a timedependent manner with peak levels observed at $72 \mathrm{~h}$ (Figure 1b). The effect of CSF1 on the invasive potential of A549 cells was determined in Matrigel assay. Figure 2 shows 

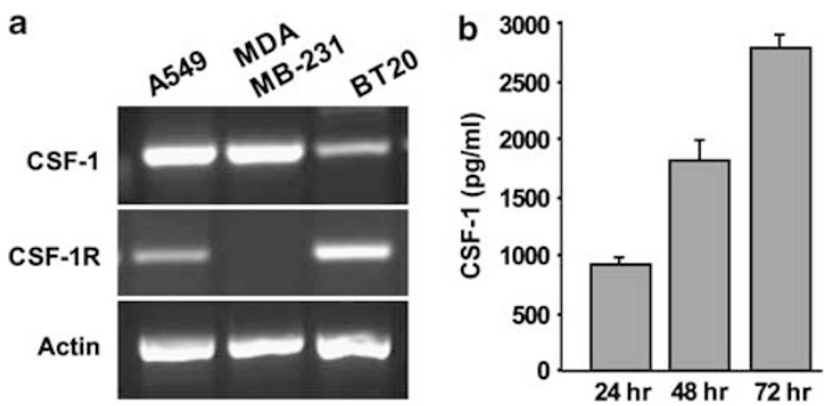

Figure 1 Expression of CSF1 mRNA and protein and CSF1R by A549-luc cells. (a) RT-PCR analysis of CSF1 and CSF1R mRNAs in A549-luc cells; human breast cancer cell lines, MDA MB-231 and BT20, were used as controls. Total RNA was isolated from cells and RT-PCR was carried out using human CSF1, CSF1R, or actin (control) primer sets. A549 cells express CSF1 and CSF1R mRNA. (b) CSF1 protein production by A549 cells. Conditioned medium was collected from A549 cell cultures at the indicated time points and assayed for human CSF1 protein using an ELISA. Mean \pm s.e., two experiments, each performed in quadruplicate wells.

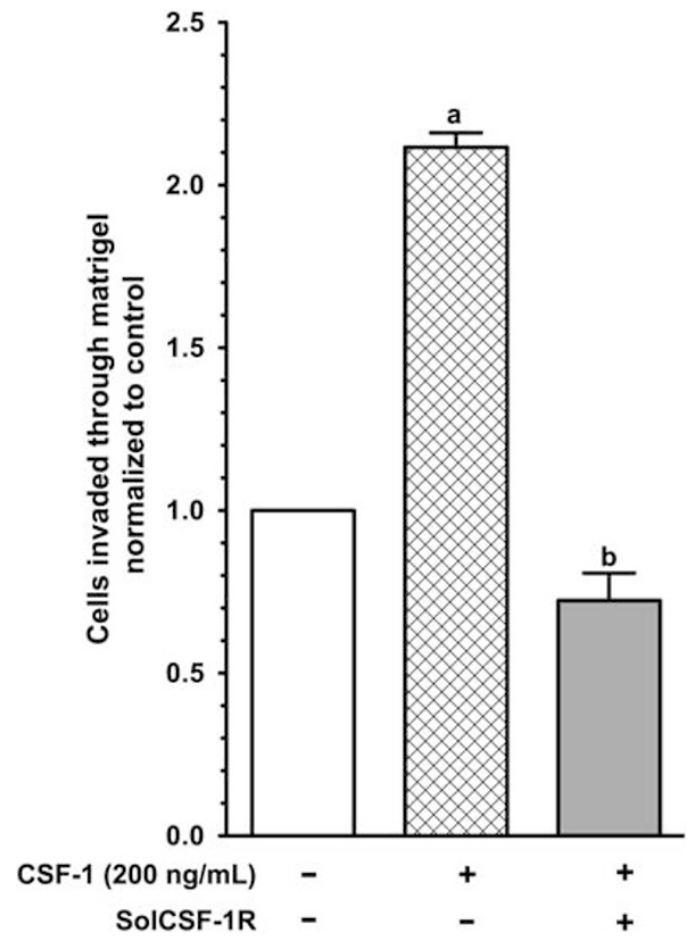

Figure 2 Effect of CSF1 on Matrigel invasion by A549-luc cells. Cells were seeded into upper inserts of Matrigel invasion chambers in SF medium. The outer wells contained SF medium with or without CSF1 $(200 \mathrm{ng} / \mathrm{ml})$. In some wells, soluble CSF1R $(2.5 \mu \mathrm{g} / \mathrm{ml})$ was added to the upper insert. After $48 \mathrm{~h}$, the non-invading cells were removed and the membrane containing the invading cells was stained with hematoxylin and eosin (H\&E) and counted under light microscopy. CSF1 stimulates A549 Matrigel invasion, whereas solCSF1R inhibits this effect. Mean \pm s.e., two experiments, each performed in triplicate wells. (a) ${ }^{* *} P<0.01$ vs untreated cultures; (b) ${ }^{*} P<0.01$ vs CSF1-treated cultures. that invasion of A549 cells increased in response to CSF1 compared with cells in SF medium. These data support previous studies showing increased invasion of A549 cells through Matrigel or amnionic basement membrane in response to CSF1. ${ }^{11,12}$ Soluble (sol)CSF1R, which blocks the interaction of CSF1 with its cognate receptor, markedly inhibited CSF1-induced invasion to levels close to that in untreated cells. ${ }^{24}$ Taken together, these findings suggest that the release of CSF1 by A549 cells may provide a strong stimulus for invasion and osteoclast-mediated bone loss in bone metastasis.

\section{Administration of A549-luc Cells Leads to Progressive Osteolytic Bone Metastasis}

To characterize a lung cancer bone metastasis model, A549luc cells were injected intracardiac in NOD/SCID mice. Mice were monitored for bone metastasis using X-ray and BLI. At 2 weeks post injection, $\mathrm{X}$-rays resembled controls and lesions were not detected. By 3.5 weeks, osteolytic bone lesions were identified in hind limbs and, at 5 weeks, large osteolytic lesions were evident in the femur, tibia, and spine (Figure 3a). Lesion number and lesion area were quantified and the bar graphs show progression of bone metastasis over 5 weeks, with a 4.5 -fold increase in lesion number and a 3 -fold increase in lesion area between 3.5 and 5 weeks (Figure $3 \mathrm{~b}$ ). BLI was performed at 3.5 and 5 weeks to evaluate the in vivo kinetics of tumor growth in the same mouse. Whole body images are shown in Figure 4a and photon counts are indicated by the color scales ranging from blue (least intense) to red (most intense). A549-luc cells produced bone metastasis in the hind limbs at 3.5 weeks, with a higher signal detected at 5 weeks in the tibia and femur. Foci of intense signal in the upper abdomen and thoracic compartment correlated with metastasis in the vertebrae and occasional ribs. Quantification of photon counts in the femur and tibia (Figure $4 \mathrm{~b}$ ) showed an exponential increase of photon emission from 3.5 to 5 weeks indicating progression of metastasis, similar to that observed by X-ray.

\section{A549-luc Mice Show High Tumor Burden and Osteoclast-mediated Bone Loss}

At 5 weeks, histological sections of hind limbs and vertebrae from A549-luc mice and age-matched sham injected mice without tumor were examined (Figures $5 \mathrm{a}$ and $\mathrm{b}$ ). Metastatic tumor on hematoxylin and eosin-stained sections corresponded to the regions of osteolysis on X-ray. Most of the trabecular bone in hind limbs and spine was replaced by tumor that filled the marrow cavity, and in some cases, the epiphysis was involved. There was full-thickness cortical bone destruction with extension of tumor into soft tissues. Increased osteoclasts were identified along the tumor/bone interface. In contrast, mice without tumor show preserved trabecular bone and bone marrow. Histomorphometric measurements shown in the bar graphs confirmed large tumor volume indicating a high tumor burden as well as 

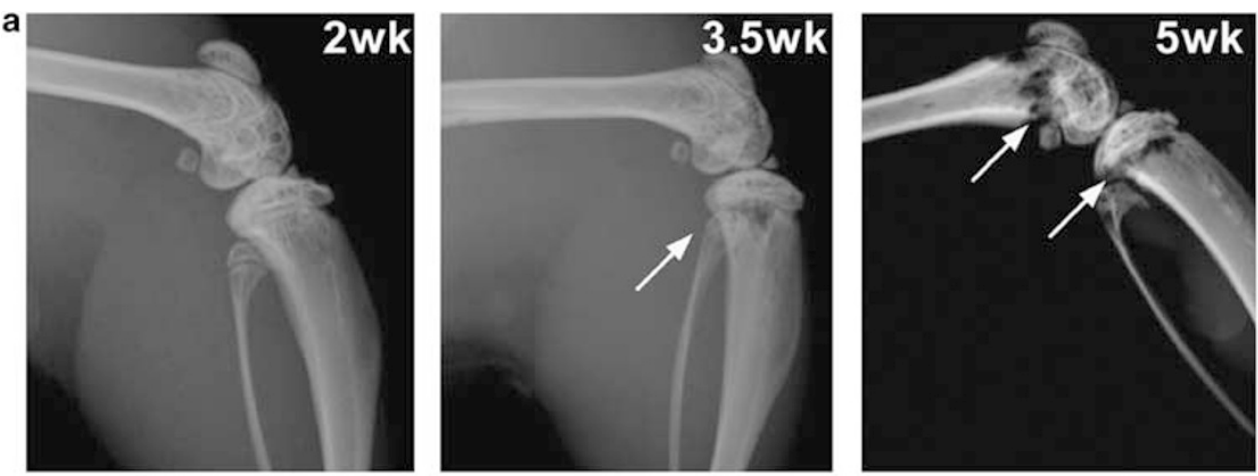

b
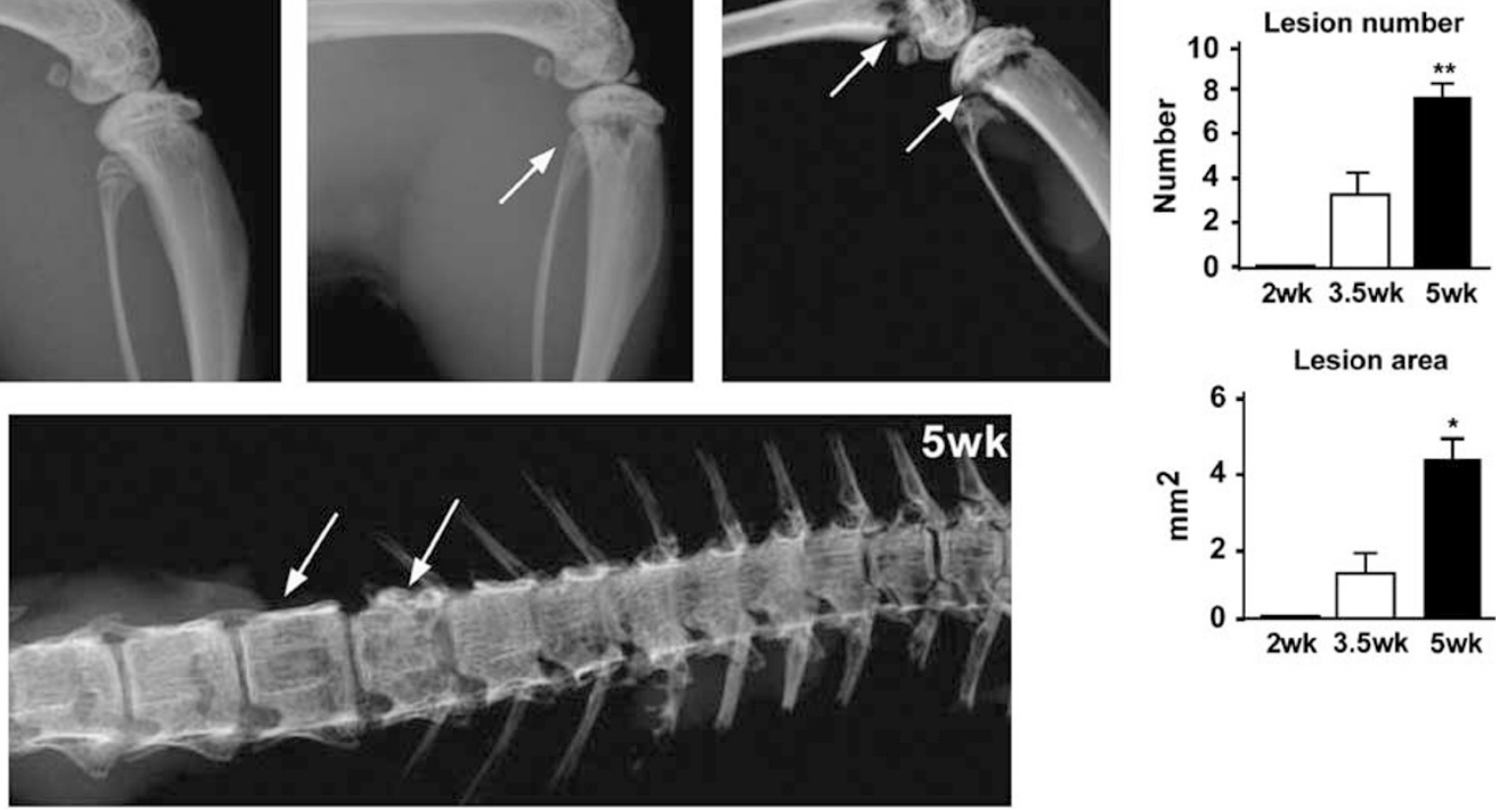

Figure 3 Comparative X-rays of hind limbs after intracardiac injection of A549-luc cells. (a) X-rays at 2, 3.5, and 5 weeks in A549-luc mice. A549-luc cells $\left(5 \times 10^{5}\right.$ in $100 \mu \mathrm{l}$ PBS) were injected intracardiac in NOD/SCID mice. After 2 weeks, lesions were not identified by X-ray. By 3.5 weeks, osteolytic lesions (arrows) were detected in hind limbs and progressively increased up to 5 weeks. X-rays at 5 weeks revealed lytic lesions in femur, tibia, and spine (arrows). (b) Quantification of lesions. At each time point, the number and area of osteolytic foci on X-rays in both hind limb bones were quantified using Bioquant image analysis system. Bar graphs show that bone metastasis increased over a 5-week period, with $\sim 4.5$-fold increase in lesion number and 3 -fold increase in lesion area between 3.5 and 5 weeks. Mean \pm s.e. $\left(n=4\right.$ mice/group), ${ }^{*} P<0.05$, ${ }^{* *} P<0.01$ vs lesions at 3.5 weeks.
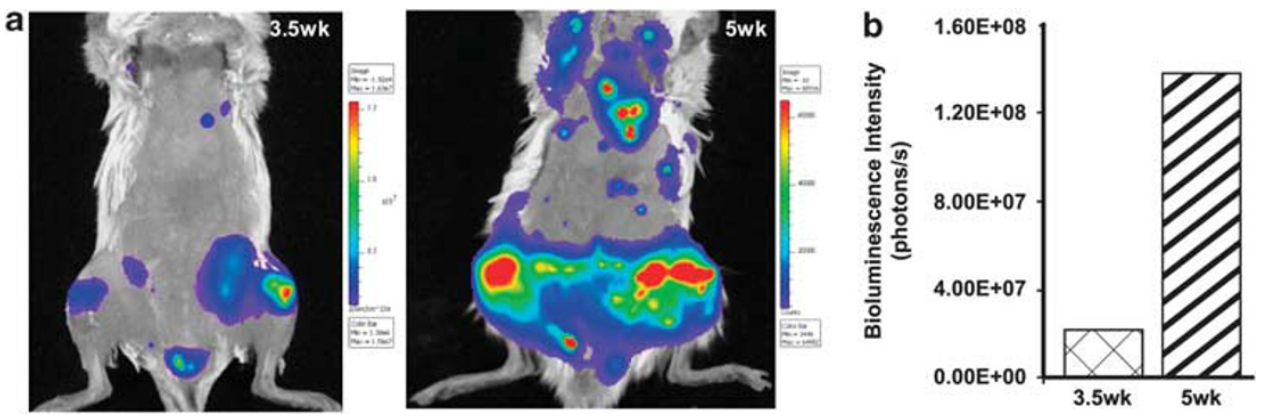

Figure 4 In vivo monitoring of bone metastasis in A549-luc mice using BLI. (a) Representative whole-body BLI at 3.5 and 5 weeks after injection of A549-luc cells. BLI started at 3.5 weeks to monitor the kinetics of tumor progression in the same animal over time. Mice were placed in the light-tight chamber of the CCD camera system (Xenogen) and photons emitted from A549-luc-expressing cells within the animal were quantified Living Image software (Xenogen). The color scales ranging from blue (least intense) to red (most intense) indicate the amount of photons emitted. A549-luc-induced bone metastasis in the hind limbs at 3.5 weeks, with a higher signal detected at 5 weeks. (b) Quantification of photon counts in femur and tibia shows an exponential increase of photon emission, indicating progression of bone metastasis between these time points.

extensive bone loss in A549-luc mice compared with nontumor-bearing mice. Osteoclast number along the tumor/ bone interface was significantly increased in A549-luc mice, whereas fewer osteoclasts were detected along normal bone trabeculae.

To assess tumor morphology in vivo, tissue sections were examined for cytokeratin (epithelial marker), CSF1, Ki-67 (proliferation marker), F4/80 (macrophages), and CD31 (vascular endothelial cells) using immunohistochemistry and apoptosis using TUNEL (Figure 5c). Tumor cells formed glandular structures and were positive for cytokeratin similar to human lung adenocarcinoma. CSF1 protein was expressed; many tumor cells were positive for Ki-67, whereas few cells were TUNEL positive, reflecting a high proliferation rate. 
a
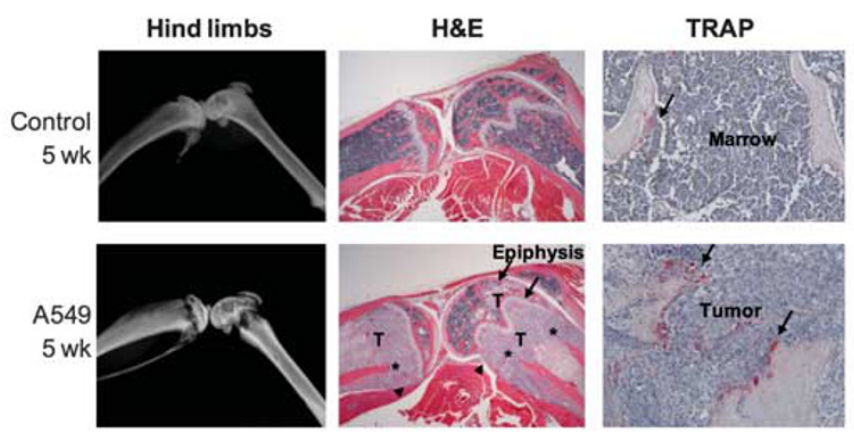
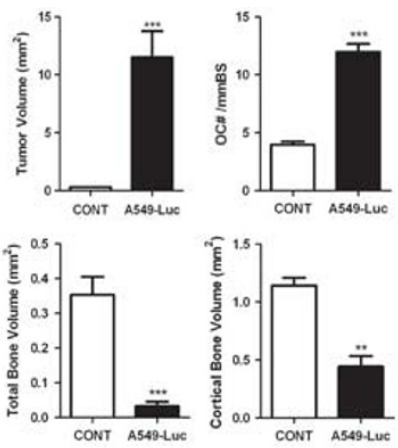

b
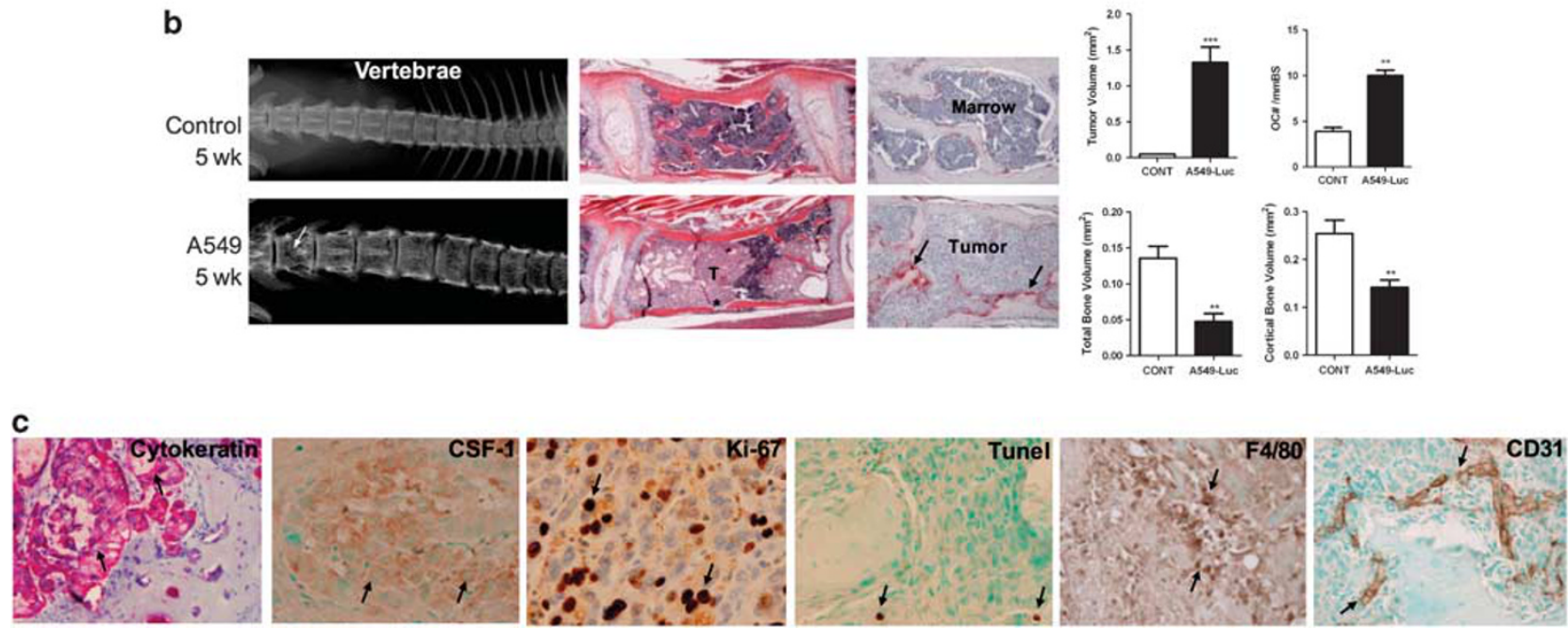

Figure 5 Bone histomorphometry in A549-luc bone metastasis. (a) X-ray, histology, and tartrate-resistant acid phosphatase (TRAP) stains of (a) hind limbs and (b) vertebrae at 5 weeks. Age-matched sham injected mice without tumor served as control (CONT). Tumor in A549-luc histology sections correspond to osteolysis on X-ray. Most of the trabecular bone is replaced by tumor (T). Cortical bone is destroyed $\left({ }^{*}\right)$ with extension of tumor into soft tissues (arrowheads); numerous osteoclasts are identified (arrows). Bar graphs show large tumor volume, increased osteoclasts, and extensive bone loss compared with non-tumor-bearing mice. Mean \pm s.e. ( $n=5$ mice/group), ${ }^{* *} P<0.01,{ }^{* *} P<0.001$ vs control mice. (c) Tumor phenotype. Tumor cells formed glands and are positive for cytokeratin (arrows, red color). CSF1 protein is expressed (arrows, brown color); many cells are Ki-67 positive (arrows, brown nuclear stain), whereas few cells are TUNEL positive (arrows, brown nuclear stain). Tumor stroma contains F4/80-positive macrophages (arrows) and angiogenesis is evident on CD31 immunostain (arrows).

A number of F4/80-positive macrophages were present in the stroma and angiogenesis was evident on CD31 immunostain. Taken together, these findings indicate that A549-luc cells localize to bone and establish metastasis. A549 mice were also examined for extraosseous tumor in all organs except the brain. A few residual tumor cells were identified in the heart from injection. Few metastases were identified in other tissues, with only small foci present in the adrenal gland and kidney, sites similar to that observed in human disease and a previous model of A549 lung cancer. ${ }^{25}$

\section{CSF1KD in A549 Cells Decreases SP fraction and Inhibits Growth}

To determine whether inhibition of CSF1 in A549 cells ameliorates bone metastasis, cells were stably transfected with a retroviral vector containing shCSF1 or empty vector control. ELISA confirmed significantly less CSF1 protein production in CSF1KD cells at $24 \mathrm{~h}(390 v s 788 \mathrm{pg} / \mathrm{ml})$ and $72 \mathrm{~h}$ compared with control cells (1884 vs $5786 \mathrm{pg} / \mathrm{ml}$ ). To assess the effect of CSF1 inhibition in A549 cells on the SP fraction, CSF1KD and control cells were then incubated with $\mathrm{DCV}$ in the presence or absence of reserpine. In the presence of reserpine, an ABCG2 transporter inhibitor, SP cells fail to efflux DCV, and are identified by diminished fluorescence (Figure 6a). ${ }^{16}$ By FACS analysis, control A549 cells were enriched with $3.2 \%$ SP cells, whereas the fraction of SP cells in CSF1KD cells was significantly decreased by 80 to $0.6 \%$ (Figures $6 \mathrm{a}$ and $\mathrm{b}$ ). The effect of CSF1KD on cell proliferation was also examined. Figure $6 \mathrm{c}$ shows increased proliferation of control cells after 5 days incubation, whereas proliferation was reduced in CSF1KD cultures. To examine tumorigenic potential in vitro, CSF1KD and control cells were analyzed in an anchorage-independent soft agar assay. CSF1KD cells showed decreased number and size of colonies in agar 

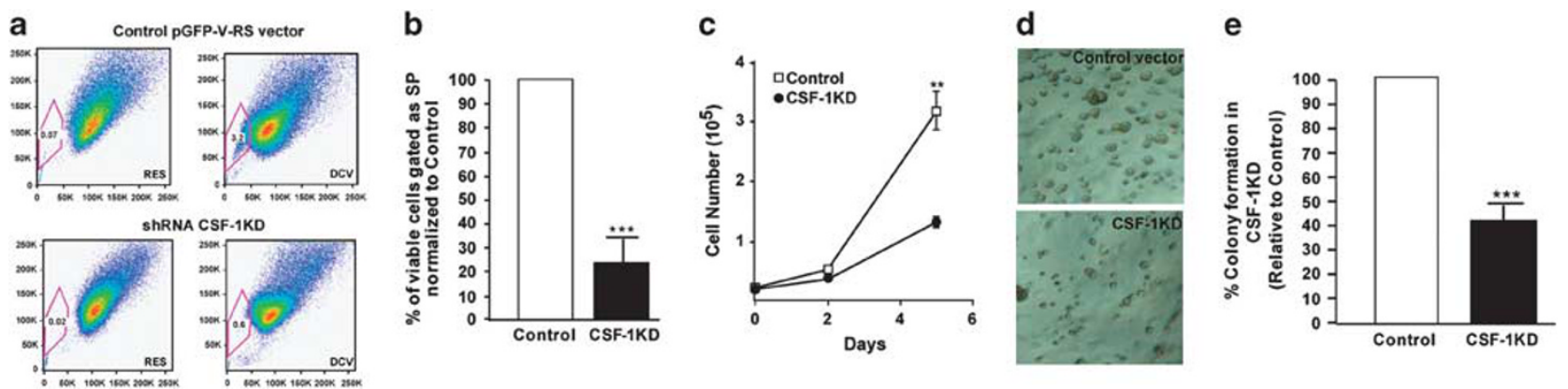

Figure 6 FACS analysis of control and shCSF1 A549 cells. (a) Representative FACS analysis of cells incubated with DCV in the presence (left panels) or absence (right panels) of reserpine (RES). DCV reagent was excited at 405 and its fluorescence was dual-wavelength analyzed. The SP gate was defined as the diminished fluorescence in the presence of reserpine (outlined region). Control A549 cells contained $\sim 3.2 \%$ SP cells, whereas the fraction of SP cells in CSF1KD cells decreased by $80 \%$ to $\sim 0.6 \%$. (b) Quantification of viable cells gated as SP normalized to the control, with control value arbitrarily set at $100 \%$. Mean \pm s.e., three experiments, each performed in triplicate. (c) Cell proliferation assay. Subconfluent cells were incubated for 2 and 5 days, trypsinized, and counted. Mean \pm s.e., two experiments, data duplicated with cells plated at $2 \times 10^{4}$ and $5 \times 10^{4}$ cells/well in triplicate, ${ }^{* *} P<0.01$. (d) Anchorage-independent growth assay. CSF1KD cells show decreased colony formation with reduced number and size of colonies in soft agar. (e) Quantification of colony formation, with control value arbitrarily set at $100 \%$. Mean \pm s.e., two experiments, data duplicated with cells seeded at $5 \times 10^{3}$ and $1 \times 10^{4}$ cells/well in quadruplicate, ${ }^{* * *} P<0.001$ vs control.

(Figure 6d) and quantification confirmed reduced colony formation compared with control (Figure 6e).

\section{Administration of CSF1KD A549 Cells Leads to Smaller Osteolytic Bone Lesions}

Mice were then injected with $1 \times 10^{5}$ control or CSF1KD A549 cells. The incidence of tumor metastasis was similar in both groups with $70-75 \%$ of mice developing tumors. At 5 weeks, control mice showed replacement of the marrow cavity by tumor and full-thickness cortical bone destruction (Figure 7a). In contrast, CSF1KD mice showed a dramatic reduction in tumor size that corresponded with smaller osteolytic bone lesions on X-ray and microCT. There was a trend for fewer CSF1KD mice to show tumor involving both hind limbs compared with controls (42\% vs $83 \%$; Figure $7 \mathrm{~b}$ ). Importantly, there was a significantly lower incidence of large tumors $>0.1 \mathrm{~mm}^{2}$ in CSF1KD mice $(10 \%)$ compared with controls $(62.5 \%$; Figure $7 \mathrm{c})$. This was associated with a reduction in percent tumor volume in CSF1KD mice (1.5\%) compared with controls (8.0\%; Figure 7d). Moreover, compared with control tumors, tumors in CSF1KD mice showed a dramatic reduction in CSF1 expression that correlated with decreased Ki-67 + tumor cell proliferation and osteoclast numbers along the tumor/bone interface (Figures $8 \mathrm{a}, \mathrm{b}$, and c). A significant difference in the number of $\mathrm{F} 4 / 80+$ macrophages and vessels between CSF1KD and control tumors was not observed (Figures 8d and e), although the possibility that these parameters differed at earlier time points cannot be excluded. Within our experimental groups, tumor size varied, which could be related to the method of cell delivery using intracardiac injection. This technique leads to metastasis within the metaphysis of long bones as in our model and growth may be influenced by local factors including blood flow rate and blood vessel architecture. ${ }^{26}$

\section{DISCUSSION}

This study characterized a lung cancer osteolytic bone metastasis model using the A549 lung adenocarcinoma cell line. The biologic effect of CSF1 in A549 cells is summarized in Figure 9. We show that A549 cells express CSF1 and its receptor. CSF1 increased A549 proliferation and invasion, indicating that A549 cells are a source and target of CSF1. In vitro, CSF1KD in A549 cells decreased CSF1protein, dramatically reduced SP cells, decreased proliferation, and soft agar colony formation, suggesting that CSF1 is a key determinant of lung CSC survival and tumor growth. In vivo, CSF1KD mice showed smaller osteolytic lesions and tumor volume compared with controls, suggesting that A549derived CSF1 is important for osteoclast-mediated bone resorption and tumor growth.

Intracardiac injection of human breast cancer or myeloma cell lines is a widely accepted technique for establishing bone metastasis in mice. ${ }^{22,27} \mathrm{Few}$ studies have taken advantage of this method to develop lung cancer bone metastasis and describe pathologic lesions. ${ }^{25}$ A human SPC-A-1BM lung adenocarcinoma cell line, administered intracardiac, was shown to metastasize by bone scintigraphy; however, histologic confirmation of tumor was not provided. ${ }^{2}$ Recently, Nguyen et al, ${ }^{28}$ using intracardiac injection of H2030 lung adenocarcinoma and PC9 cell lines, showed that Wnt/TCF signaling mediate lung cancer bone metastasis, although osteoclast analysis was not shown. With regard to A549 cells, testing of cancer therapeutics has been performed in xenografts where cells are injected subcutaneously. ${ }^{29}$ Tail vein injection of A549 cells rarely resulted in metastatic lesions. ${ }^{30}$ When A549 cells were administered intracardiac, mice developed bone metastasis; however, tumor morphology and bone histomorphometry were not analyzed. ${ }^{31}$ Freeley et $a b^{32}$ performed direct intratibial injections of 
a
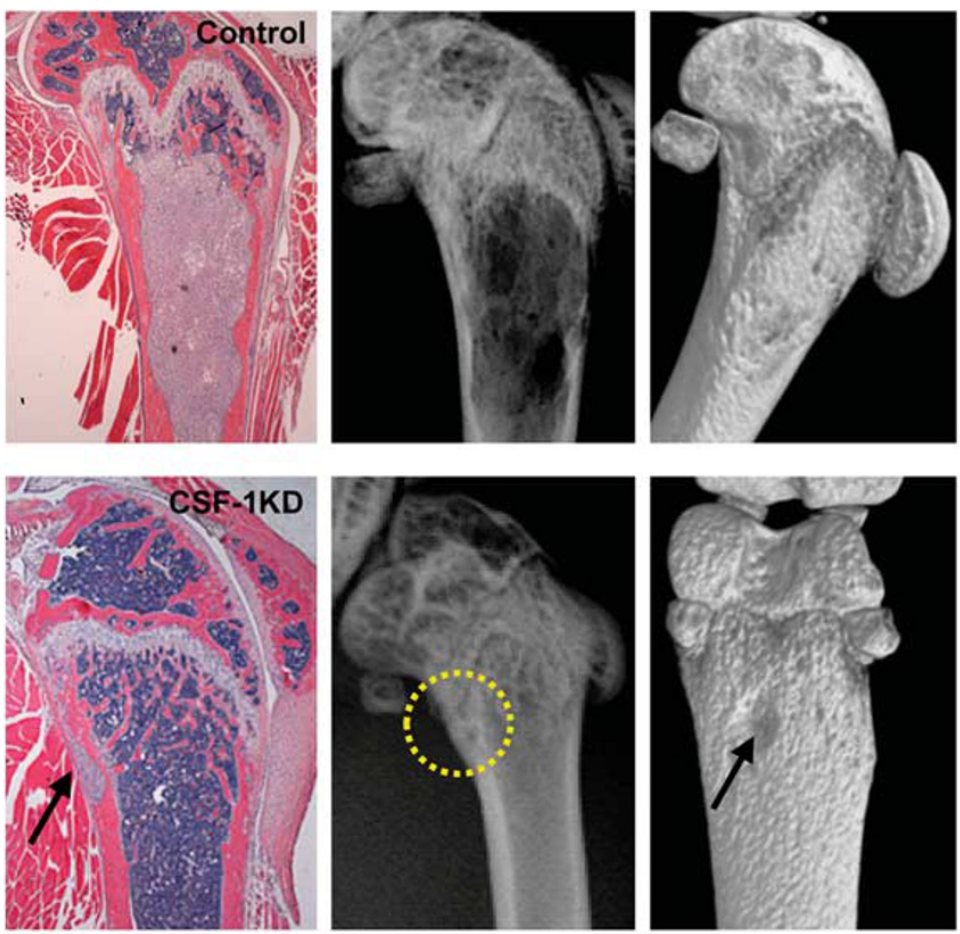

b $\%$ Both legs with tumor

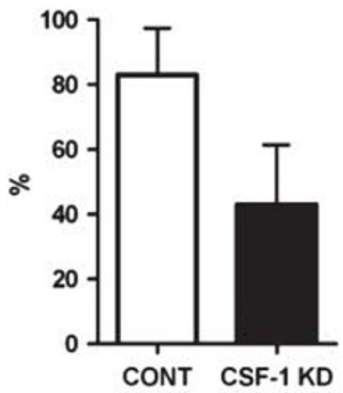

C Percent tumors $>0.1 \mathrm{~mm}^{2}$

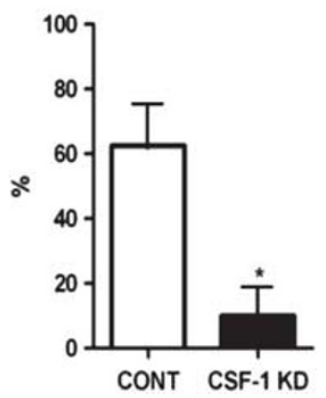

d Tumor vol/Tissue vol

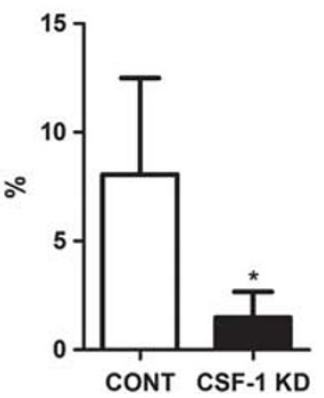

Figure 7 Effect of CSF1KD in A549 cells on bone metastasis. (a) Mice were injected with $1 \times 10^{5}$ control or CSF1KD A549 and bone lesions were analyzed by histology (left panels), X-ray (middle panels), and microCT (right panels). The incidence of tumor metastasis was similar in both groups. At 5 weeks, controls show replacement of the marrow cavity by tumor and full-thickness cortical bone destruction. In contrast, CSF1KD mice show a dramatic reduction in tumor size (left lower panel, arrow) that corresponded with smaller osteolytic lesions on X-ray (middle lower panel, dotted circle) and microCT (right lower panel, arrow). (b) There is a trend for fewer CSF1KD mice (42\%) to show tumor involving both hind limbs compared with controls (83\%). (c) Bar graph shows a lower incidence of large tumors $>0.1 \mathrm{~mm}^{2}$ in CSF1KD mice (10\%) compared with controls (62.5\%). Mean \pm s.e. ( $n=8-10$ mice/group), ${ }^{*} P<0.05$ vs control mice. (d) Bar graph shows decreased percent tumor volume in CSF1KD mice (1.5\%) compared with controls (8.0\%). Mean \pm s.e. $\left(n=6-8\right.$ mice/group), ${ }^{*} P=0.02$ control vs CSF1KD mice by a trimmed non-parametric test.

A549 cells; mice developed mixed osteolytic and osteoblastic lesions that were inhibited by overexpressing noggin in the cells and co-administration of RANKL-Fc. In contrast to the latter model, our A549 model resembles the human counterpart of the disease that is often associated with multiple rather than a single metastatic lesion. The onset of bone lesions in A549-luc mice was rapid; lesions were lytic and localized to the femur and spine, similar to those in humans. ${ }^{2,3}$ Tumors recapitulated adenocarcinoma morphology with a high proliferation rate and showed osteoclasts along the tumor bone interface with marked trabecular and cortical bone loss. A significant osteoblastic component was not observed in our study, which could be related to the mouse strain, age of mice, or route of A549 cell administration.

Studies in patients and in experimental models suggest that the development of bone metastasis is multifactorial and related to osteoclast activation, expansion of CSCs, and tumor-stromal interaction. Osteoclasts are identified along the tumor/bone interface in humans and rodent models. Studies indicate that osteoclasts are activated by cancer cells via the release of molecules such as PTHrP and RANKL and mediate osteolysis. ${ }^{33,34}$ It is thought that osteoclasts have an 


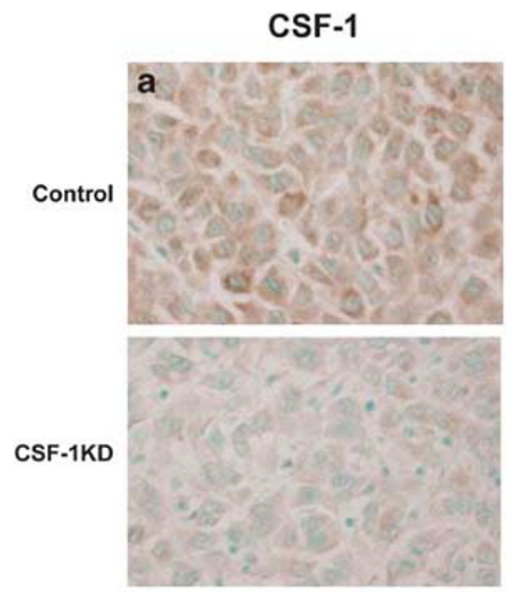

IOD of CSF-1 intensity

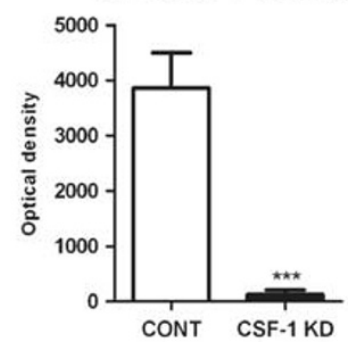

$\mathrm{Ki}-67$
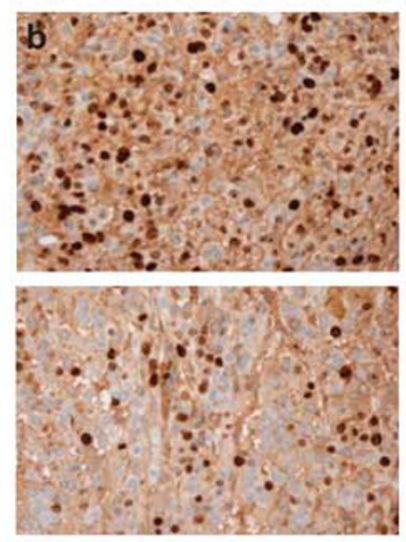

$\% \mathrm{Ki}-67$ positive cells

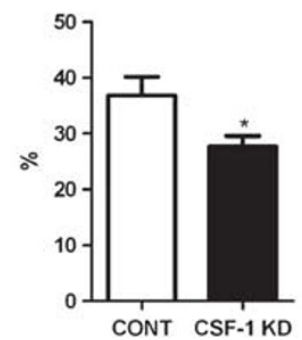

TRAP

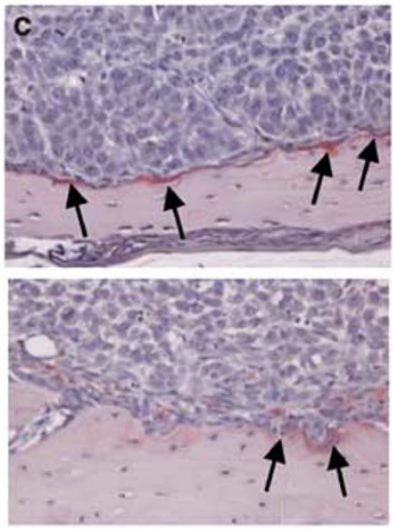

Osteoclast No.

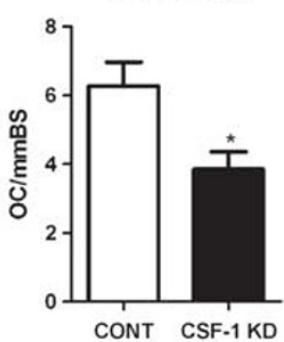

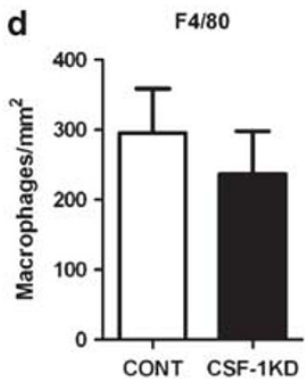

e Vessels $/ \mathrm{mm}^{2}$ tumor

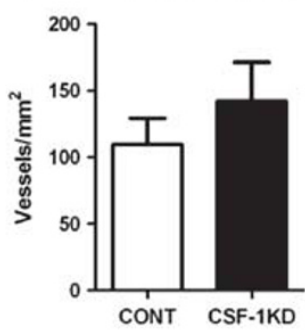

Figure 8 Analysis of tumor phenotype in control and CSF1KD mice. Upper panel: (a) CSF1-, (b) Ki67-, and (c) tartrate-resistant acid phosphatase (TRAP)stained histologic sections. Control tumors show CSF1 expression that is associated with Ki-67-positive proliferating cells and osteoclastic bone resorption (arrows). Reduced CSF1 expression is identified in CSF1KD tumors and correlates with decreased tumor proliferation and osteoclastic activity. Lower panel: (a-c) Quantification of cellular markers. Intensity of CSF1 protein on immunostain, percent positive Ki-67 cells, and osteoclast numbers along tumor/bone interface are significantly reduced in CSF1KD tumors compared with control tumors. (d-e) Quantification of F4/80 + macrophages and vessels $/ \mathrm{mm}^{2}$ tumor. No significant difference in the number of F4/80 macrophages and vessels between CSF1KD and controls tumors was observed. Mean \pm s.e. ( $n=8-10$ mice/group), ${ }^{* *} P<0.001,{ }^{*} P<0.05$ control vs CSF1KD tumors.

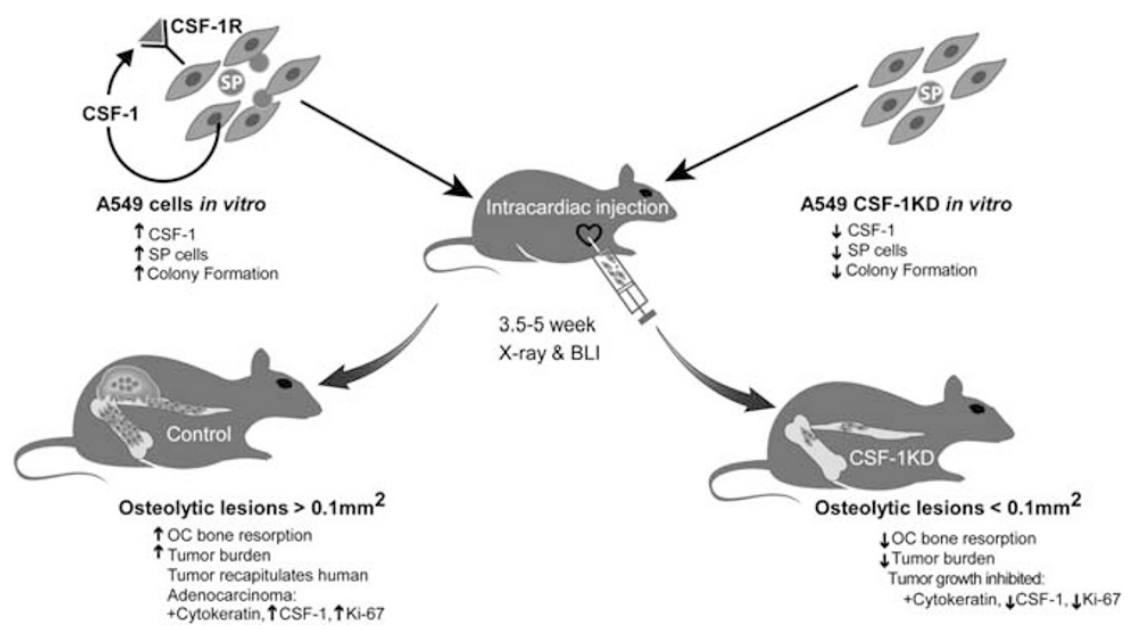

Figure 9 Effect of CSF1KD in A549 cells on bone metastasis. In vitro, knockdown of CSF1 in A549 cells decreased CSF1 and reduced the fraction of SP cells and soft agar colony formation. In vivo, CSF1KD mice showed smaller osteolytic lesions compared with control mice. Tumors in CSF1KD mice showed a dramatic reduction in CSF1 expression that correlated with decreased Ki-67 + tumor cell proliferation and osteoclast (OC) numbers along with the tumor/bone interface.

initiating and integral role in stimulating bone metastatic tumor growth and progression in the marrow cavity. This is supported by work showing that inhibitors of osteoclastic bone resorption including anti-PTHrP antibody, an antibody to RANKL, osteoprotegerin, and bisphosphonates reduce bone metastasis of breast cancer. ${ }^{22,35-37}$ In bone metastasis of 
breast cancer, there is a vicious cycle wherein bone-residing tumor cells stimulate osteoclast-mediated bone destruction and bone-stored factors, including TGF- $\beta$ and BMPs released from the resorbed bone, promote tumor growth.

Our results indicate a role for CSF1 in tumorigenesis and osteolysis. Release of CSF1 from A549 cells may act in an autocrine manner to enhance proliferation and invasion of tumor as well as in a paracrine manner to increase osteoclast formation. A vicious cycle may also ensue as CSF1 is also a bone-stored factor and release from resorbed bone may further promote tumor expansion. ${ }^{38}$ Indeed, release of CSF1 by tumor cells in A549-luc mice was associated with increased osteoclasts along the tumor/bone interface and progressive increase in osteolytic lesions from 3.5 to 5 weeks. In addition to osteolysis, cancer stem-like cells within the tumor may sustain the relentless growth of the mass. ${ }^{14,15,39}$ Recent studies indicate that human lung cancers, as in other cancers, have their own stem-like cells. ${ }^{39}$ Cancer stem-like cells can be identified based on their functionality, notably the ability to efflux the Hoechst 33342 dye or Dye cycle Violet, which on FACS analysis appears as a distinct population of cells on the side of the FACS profile; hence, the name 'SP'. ${ }^{16}$ Using reserpine, which inhibits the ABCG2 transporter in SP cells to efflux the DCV, we identified a small fraction of SP cells (2-6\%) in cultured A549 cells.

Studies indicate that CSF1 enhances tumor progression; however, its effect in lung cancer has not been well defined. ${ }^{40}$ Mice with ovarian cancer cells expressing high levels of CSF1 showed widespread visceral and distant metastasis compared to mice with control cells. ${ }^{9}$ Overexpression of CSF1 in the mammary cancer-susceptible polyoma middle T antigen mice resulted in acceleration of malignant progression and metastasis. ${ }^{10}$ Conversely, the absence of CSF1 in op/op mice in the polyoma middle $\mathrm{T}$ antigen background retarded mammary tumor progression and metastasis. ${ }^{41}$ In mice with subcutaneous breast cancer xenografts, treatment with antiCSF1 antibody alone reduced tumor growth and, in combination with chemotherapy, reversed chemoresistance and enhanced survival. ${ }^{42}$ To delineate the effect of CSF1 deficiency on A549 tumor metastasis, we generated stable A549 cells expressing either shCSF1 (KD) or empty vector control. In vitro analysis confirmed that shCSF1 significantly decreased CSF1 protein levels compared with control. Moreover, CSF1KD cells showed a dramatic reduction in the fraction of SP cells, proliferation of tumor cells, and anchorage-independent colony formation in soft agar.

When mice were injected with control or CSF1KD A549 cells, the incidence of tumor metastasis in both groups was similar; however, fewer CSF1KD mice had metastasis in both hind limbs. CSF1KD tumors showed reduced CSF1 expression that was associated with decreased $\mathrm{Ki}-67+$ proliferating cells. Osteoclasts along with the tumor/bone interface were substantially reduced in CSF1KD mice and correlated with smaller lytic lesions detected on X-ray and microCT. Importantly, there was a low incidence of large tumors $>0.1 \mathrm{~mm}^{2}$ in
CSF1KD mice compared with control mice (10\% vs $62.5 \%)$ that was associated with reduced tumor volume. Although we cannot exclude paracrine effects of CSF1 from resident cells within the bone microenvironment, these findings indicate an important role for A549-derived CSF1 in tumor growth. Interestingly, in breast and colon cancer, CSF1 is also implicated in enhancing tumor progression by its effect on macrophages. ${ }^{40-43}$ CSF1 stimulates macrophage influx and induces VEGF in macrophages that, in turn, stimulate angiogenesis. ${ }^{40}$ In our study, F4/80 macrophages and angiogenesis in CSF1KD tumors were not significantly different from that observed in control tumors, possibly related to a compensatory effect of CSF1 produced by cells in the microenvironment. Whether complete KD of CSF1 in A549 tumor cells would impair macrophage influx and angiogenesis would be of interest. In our model, decreased osteolytic activity in CSF1KD mice is likely due to decreased CSF1 production by the tumor cells. It is tempting to speculate that decreased cancer stem-like cells and osteoclastogenesis contributed to smaller osteolytic lesions and decreased tumor progression in CSF1KD mice.

In summary, our data suggest that CSF1 is a multifunctional molecule in lung cancer bone metastasis and regulates tumor cell proliferation, CSCs, and osteoclastic bone resorption. The A549 metastatic model provides a valuable pre-clinical model for developing strategies to reduce lung cancer bone metastasis. Inhibition of CSF1 may provide a useful therapeutic approach for preventing tumor invasion and complications of metastatic bone disease.

\section{ACKNOWLEDGMENTS}

We thank Roberto Fajardo, PhD, and James Schmitz at RAYO, the Daniel W Carlisle Center for Bone and Mineral Imaging at UTHSCSA for their assistance in analyzing the skeletal tissues. We also thank the Flow Cytometry Shared Resource Facility at UTHSCSA, which is supported by $\mathrm{NIH}$ NCI P30CA54174 (CTRC at UTHSCSA) for fluorescence-activated cell-sorting analysis. This work was supported by the Biomedical Research Foundation of South Texas (SA-W, FT).

\section{DISCLOSURE/CONFLICT OF INTEREST}

The authors declare no conflict of interest.

1. Weir HK, Thun MJ, Hankey BF, et al. Annual report to the nation on the status of cancer, 1975-2000, featuring the uses of surveillance data for cancer prevention and control. J Natl Cancer Inst 2003;95:1276-1299.

2. Yang S, Dong Q, Yao M, et al. Establishment of an experimental human lung adenocarcinoma cell line SPCA-1BM with high bone metastases potency by $99 \mathrm{mTc}-\mathrm{MDP}$ bone scintigraphy. Nuclear Med Biol 2009;36:313-321.

3. Sugiura $H$, Yamada $K$, Sugiura $T$, et al. Predictors of survival in patients with bone metastasis of lung cancer. Clin Orthop Relat Res 2008;466:729-736.

4. Tanaka S, Takahashi N, Udagawa N, et al. Macrophage colonystimulating factor is indispensable for both proliferation and differentiation of osteoclast progenitors. J Clin Invest 1993;91: 257-263.

5. Fuller K, Owens JM, Jagger CJ, et al. Macrophage colony-stimulating factor stimulates survival and chemotactic behavior in isolated osteoclasts. J Exp Med 1993;178:1733-1744.

6. Kacinski BM. CSF1 and its receptor in breast carcinomas and neoplasms of the female reproductive tract. Mol Reprod Dev 1997; 46:71-74. 
7. Ide $\mathrm{H}$, Seligson $\mathrm{DB}$, Memarzadeh $\mathrm{S}$, et al. Expression of colonystimulating factor 1 receptor during prostate development and prostate cancer progression. Proc Natl Acad Sci USA 2002;99: 14404-14409.

8. Toy EP, Azodi M, Folk NL, et al. Enhanced ovarian cancer tumorigenesis and metastasis by the macrophage colony-stimulating factor. Neoplasia 2009;11:136-144.

9. Kirma N, Luthra R, Jones J, et al. Overexpression of the colony stimulating factor (CSF1) and/or its receptor c-fms in mammary glands of transgenic mice results in hyperplasia and tumor formation. Cancer Res 2004;64:4162-4170.

10. Kaminska J, Kowalska M, Kotowicz B, et al. Pretreatment serum levels of cytokines and cytokine receptors in patients with non-small cell lung cancer, and correlations with clinicopathological features and prognosis. Oncology 2006;70:115-125.

11. Uemura $\mathrm{Y}$, Kobayashi M, Nakata $\mathrm{H}$, et al. Effects of GM-CSF and M-CSF1 on tumor progression of lung cancer. Int J Mol Med 2006;18:365-373.

12. Filderman $\mathrm{AE}$, Bruckner $\mathrm{A}$, Kacinski $\mathrm{BM}$, et al. Macrophage colonystimulating factor (CSF1) enhances invasiveness in CSF1 receptorpositive carcinoma cell lines. Cancer Res 1992;52:3661-3666.

13. Tsuchimoto D, Tojo A, Asano S. A mechanism of transcriptional regulation of the CSF1 gene by interferon-gamma. Immunol Invest 2004;33:397-405.

14. Yagui-Beltran A, He B, Jablons DM. The role of cancer stem cells in neoplasia of the lung: past, present and future. Clin Transl Oncol 2008;10:719-725.

15. Hung JY. Tumor stem cells and metastasis. In: Keshamouni V Arenberg D, Kalemkerian G (eds). Novel Biological Mechanisms and Impact on Clinical Practice: Lung Cancer Metastasis. Springer Science and Business Media, LLC: New York, 2009.

16. Ho MM, Ng AV, Lam S, et al. Side population in human lung cancer cell lines and tumors is enriched with stem-like cancer cells. Cancer Res 2007;67:4827-4833.

17. Meuwissen R, Berns A. Mouse models for human lung cancer. Genes Dev 2005:19:643-664.

18. Harris SE, Macdougall M, Horn D, et al. Meox2Cre-mediated disruption of CSF1 leads to osteopetrosis and osteocyte defects. Bone 2012; 50:42-53.

19. Ryan GR, Dia X-M, Dominguez MG, et al. Rescue of the colonystimulating factor 1 (CSF1-nullizygous mouse (Csf1op/Csf1op) phenotype with a CSF1 transgene and identification of sites of local CSF1 synthesis. Blood 2001;98:74-84.

20. Gallet M, Mentaverri R, Sevenet N, et al. Ability of breast cancer cell lines to stimulate bone resorbing activity of mature osteoclasts correlates with an anti-apoptotic effect mediated by macrophage colony stimulating factor. Apoptosis 2006:11:1909-1921.

21. Dikmen ZG, Gellert GC, Jackson S, et al. In vivo inhibition of lung cancer by GRN163L: a novel human telomerase inhibitor. Cancer Res 2005;65:7866-7873.

22. Guise TA, Yin JJ, Taylor SD, et al. Evidence of a causal role of parathyroid hormone-related protein in the pathogenesis of human breast cancer-mediated osteolysis. J Clin Invest 1996:98:1544-1549.

23. Nogawa M, Yuasa T, Kimura S, et al. Monitoring luciferase labeled cancer cell growth and metastasis in different in vivo models. Cancer Lett 2005;217:243-253.

24. Gowardhan B, West AF, Robson $\mathrm{CN}$, et al. Adenovirus-mediated expression of soluble fibroblast growth factor receptor inhibits in vitro growth of prostate DU145 cells. Prostate 2004;61:50-59.
25. Luis-Ravelo D, Anton I, Vicent S, et al. Tumor-stromal interactions of the bone microenvironment: in vitro findings and potential in vivo relevance in metastatic lung cancer models. Clin Exp Metastasis 2011;28:779-791.

26. Rosol TJ, Tannehill-Gregg SH, LeRoy BE, et al. Animal models of bone metastasis. Cancer Treat Res 2004;361:47-81.

27. Mundy G. Preclinical models of bone metastasis. Semin Oncol 2001;28:2-8.

28. Nguyen DX, Chiang AC, Zhang $X$, et al. WNT/TCF signaling through LEF1 and HOXB9 mediates lung adenocarcinoma metastasis. Cell 2009;138:1-12.

29. Coxon A, Ziegler B, Kaufman S, et al. Antitumor activity of motesanib alone and in combination with cisplatin or docetaxel in multiple human non-small-cell lung cancer xenograft models. Mol Cancer 2012;11:70-78.

30. Zhang J, Niu XM, Liao ML, et al. Discrepancy of biologic behavio influenced by bone marrow derived cells in lung cancer. Oncol Rep 2010;24:1185-1192.

31. Catena R, Luis-Ravelo D, Anton I, et al. PDGFR signaling blockade in marrow stroma impairs lung cancer bone metastasis. Cancer Res 2010;71:164-174.

32. Freeley $\mathrm{BT}$, Liu NQ, Conduah $\mathrm{AH}$, et al. Mixed metastatic lung cancer lesions in bone are inhibited by noggin overexpression and Rank:Fc administration. J Bone Miner Res 2006;21:1571-1580.

33. Clines GA, Guise TA. Molecular mechanisms and treatment of bone metastasis. Exp Rev Mol Med 2008;10:1-15.

34. Mundy GR. Metastasis to bone: causes, consequences and therapeutic opportunities. Nat Rev Cancer 2002;2:584-593.

35. Hiraga T, Williams PJ, Mundy GR, et al. The bisphosphonate ibandronate promotes apoptosis in MDA MB-231 human breast cancer cells in bone metastases. Cancer Res 2001;61:4418-4424.

36. Chanda D, Isayeva T, Kumar S, et al. Systemic osteoprotegerin gene therapy restores tumor-induced bone loss in a therapeutic model of breast cancer bone metastasis. Mol Ther 2008;16:871-878.

37. Lipton A, Steger GG, Figueroa J, et al. Randomized active-controlled phase II study of denosumab efficacy and safety in patients with breast cancer-related bone metastasis. J Clin Oncol 2007;25: 4431-4437.

38. Ohtsuki T, Suzu S, Hatake K, et al. A proteoglycan form of macrophage colony-stimulating factor that binds to bone-derived collagens can be extracted from bone matrix. Biochem Biophys Res Commun 1993;190: 215-222.

39. Castellon EA, Valenzuela R, Lillo J, et al. Molecular signature of cancer stem cells isolated from prostate carcinoma and expression of stem markers in different Gleason grades and metastasis. Biol Res 2012; 45:297-305.

40. Aharinejad $S$, Paulus $P$, Sioud $M$, et al. Colony stimulating factor- 1 blockade by antisense oligonucleotides and small interfering RNAs suppresses the growth of human mammary tumor xenografts in mice. Cancer Res 2004;64:5378-5384.

41. Lin EY, Gouon-Evans V, Nguyen AV, et al. The macrophage growth factor CSF1 in mammary gland development and tumor progression. J Mammary Gland Biol Neoplasia 2002;7:147-162.

42. Paulus $P$, Stanley ER, Schafer $R$, et al. Colony-stimulating factor-1 antibody reverses chemoresistance in human MCF-7 breast cancer xenografts. Cancer Res 2006;66:4349-4356.

43. Aharinejad $S$, Sioud $M$, Lucus $T$, et al. Target validation using RNA interference in solid tumors. Methods Mol Biol 2007;361:227-238. 